\title{
CONVEX COMBINATIONS OF MARKOV TRANSITION FUNCTIONS ${ }^{1}$
}

\author{
S. R. FOGUEL
}

1. Introduction. Let $(\Omega, \Sigma, \lambda)$ be a measure space with $\lambda(\Omega)=1$. Consider the set $\mathfrak{A}$ of all the operators, $P$, on $L_{2}(\Omega, \Sigma, \lambda)$ such that:

(1.1) $\|P\|=1$

(1.2) $P 1=1$

(1.3) if $f \in L_{2}$ and $f \geqq 0$ then $P f \geqq 0$ a.e.

These operators are given by a Markov transition function for which $\lambda$ is an invariant measure.

The set $\mathfrak{A}$ is a weakly closed convex set and is selfadjoint.

We shall study several notions that are related to "mixing" properties and will show:

a convex combination mixes better than its generators.

Many of the results can be phrased, and their proofs are identical, for general contractions on a Hilbert space. This will be mentioned without details to avoid repetitions.

Let us repeat the following definitions from [1]. For each $P \in \mathfrak{A}$ :

(1.4) $K(P)=\left\{f: f \in L_{2}(\lambda),\left\|P^{n} f\right\|=\left\|P^{*_{n}} f\right\|=\|f\|, n=1,2, \cdots\right\}$.

(1.5) $H_{0}(P)=\left\{f: f \in L_{2}(\lambda)\right.$, weak $\left.\lim P^{n} f=0\right\}$.

(1.6) $H_{1}(P)=H_{0}(P)^{\perp}$.

It is proved in [1, Theorems 2 and 4$]$ that these are subspaces and $H_{1}(P) \subset K(P)$ and

(1.7) $K(P)=L_{2}\left(\Omega_{1}, \Sigma_{1}, \lambda\right) \Sigma_{1} \subset \Sigma$ and $U \Sigma_{1}=\Omega_{1}$.

Except for (1.7) all these definitions and results are valid for general contractions on a Hilbert space.

Our aim will be to find how "small" is $H_{1}(P)$. Thus if $\Sigma_{1}$ is atomic the "Limit Theorem" [ 1 , Theorem 8$]$ holds and if $H_{1}=$ constant functions, then $P$ is strongly mixing.

The notions of ergodic and strongly mixing operators are defined in $[3]$.

2. Convex combination of finitely many Markov transition functions.

Theorem 1. Let $P_{1}, \cdots, P_{m}$ belong to $\mathfrak{A}$ and $P=\Sigma \alpha_{i} P_{i}$ where $\alpha_{i}>0$ and $\Sigma \alpha_{i}=1$. Then

Received by the editors November 18, 1966.

${ }_{1}$ The research reported in this document has been sponsored by the Office of Scientific Research, OAR under Grant AF EOAR 66-18 through the European Office of Aerospace Research, United States Air Force and by NSF-GP 3906 and NSF-GP 4033 in the University of Minnesota, Minneapolis. 


$$
K(P)=\bigcap_{i=1}^{m} K\left(P_{i}\right) \cap \bigcap_{n=1}^{\infty}\left\{f: P_{i}^{n} f=P_{j}^{n} f, P_{i}^{* n} f=P_{j}^{* n} f\right.
$$

$$
\text { for all } 1 \leqq i, j \leqq m\} .
$$

Proof. It is enough to consider the case $m=2$ since a convex combination of $P_{1}, \cdots, P_{m}$ can be represented as a convex combination of $P_{1}$ and $Q$ where $Q$ is a convex combination of $P_{2}, \cdots, P_{m}$.

Let $f \in K(P)$ then

$$
\begin{aligned}
\|f\|=\left\|\left[\alpha P_{1}+(1-\alpha) P_{2}\right]^{n} f\right\| & \leqq \Sigma \alpha^{j}(1-\alpha)^{n-j}\left\|P_{1}^{h_{1}} P_{2}^{k_{1}} \cdots P_{1}^{h_{n}} P_{2}^{k_{n}} f\right\| \\
& \leqq \Sigma \alpha^{j}(1-\alpha)^{n-j}\|f\|=\|f\|
\end{aligned}
$$

where $h_{i} k_{i}$ assume the values 0,1 and $\Sigma h_{i}=j, \Sigma k_{i}=n-j$ and the sum is taken over all such choices when $j$ ranges between 0 and $n$. Thus each of the terms in the sum must have the norm $\|f\|$ (since the operators $P_{1}$ and $P_{2}$ are contractions). In particular $\left\|P_{1}^{n} f\right\|=\left\|P_{2}^{n} f\right\|$ $=\|f\|$. Also a Hilbert space is strictly convex and equality can occur in the triangular inequality only when the terms are proportional. Thus $P_{1}^{n} f=\gamma P_{2}^{n} f$ for some $\gamma \geqq 0$ and since they have the same norms $P_{1}^{n} f=P_{2}^{n} f$. Finally if $f \in K(P)$ then $\left\|P^{*_{n}} f\right\|=\|f\|$ and the same argument would apply to $P_{1}^{*}$ and $P_{2}^{*}$.

Conversely let $P_{1}^{n} f=P_{2}^{n} f, P_{1}^{*_{n}} f=P_{2}^{*_{n}} f$ and $f \in K\left(P_{1}\right)$. Then

$$
P_{1}^{h_{1}} P_{2}^{k_{1}} \cdots P_{1}^{h_{n}} P_{2}^{k_{n}} f=P_{1}^{h_{1}} P_{2}^{k_{1}} \cdots P_{1}^{h_{n}} P_{1}^{k_{n}} f=P_{1}^{h_{1}} P_{2}^{k_{1}} \cdots P_{2}^{h_{n}+k_{n}} f
$$

continuing in this way we shall get $P_{1}^{n} f$ and thus $P^{n} f=P_{1}^{n} f$. Now since $f \in K\left(P_{1}\right),\left\|P^{n} f\right\|=\left\|P_{1}^{n} f\right\|=\|f\|$. The result for $P^{*}$ is analogous.

TheOREM 2. Let $P_{1} \cdots P_{m} \in \mathfrak{A}$ and $P=\Sigma \alpha_{i} P_{i}$ where $\alpha_{i}>0$ and $\Sigma \alpha_{i}=1$. Then

$$
\begin{aligned}
H_{1}(P)=\bigcap_{i=1}^{m} H_{1}\left(P_{i}\right) \cap \bigcap_{n=1}^{\infty}\left\{f: P_{i}^{n} f=P_{j}^{n}, P_{i}^{* n} f=P_{j}^{* n} f\right. \\
\quad \text { for all } 1 \leqq i, j \leqq m\} .
\end{aligned}
$$

Proof. If $f \in H_{1}(P)$ then $f \in K(P)$ hence $P^{n} f=P_{i}^{n} f$ for every $1 \leqq i \leqq m$. By $\left[2\right.$, Theorem 3.1] $f \in H_{1}\left(P_{i}\right)$. The converse is proved in the same way.

Note that Theorems 1 and 2 hold for any contraction in a Hilbert space.

From (2.1) follows that, under the assumptions of Theorem 1, $\Sigma_{1}(P) \subset \bigcap_{i=1}^{m} \Sigma_{1}\left(P_{i}\right)$. Hence if at least one of the fields $\Sigma_{1}\left(P_{i}\right)$ is atomic, then so is $\Sigma_{1}(P)$. 
From (2.2) follows, under the assumptions of Theorem 2, that if at least one of the operators $P_{i}$ is strongly mixing so is $P$. Thus if $P_{1}$ is strongly mixing and $P_{2}$ any operator in $\mathfrak{A}$ then $\alpha P_{1}+(1-\alpha) P_{2}$ is strongly mixing for any choice of $0<\alpha<1$. Thus $P_{2}$ can be approximated in norm by strongly mixing operators. Let $Q$ be an invertible ergodic transformation in $\mathfrak{A}$. By the Second Category Theorem [3, p. 78] such transformations exist. Put $P=\frac{1}{2}\left(Q+Q^{2}\right)$. Now if $f \in K(P)$ then $Q f=Q^{2} f$ by Theorem 1 hence $f=Q f$ and $f$ is a constant. This shows that there is at least one strongly mixing operator in $\mathfrak{A}$ and hence a dense set of $\mathfrak{A}$. Notice that we did not show the existence of strongly mixing transformations but only operators in $\mathfrak{A}$.

Let us conclude this chapter with the following remark: Let $P$ and $P_{1}$ belong to $\mathfrak{A}$. There exists an operator $P_{2}$ in $\mathfrak{A}$ such that $P$ is a convex combination of $P_{1}$ and $P_{2}$ iff for some $0<\alpha<1 \quad P f \geqq \alpha P_{1} f$ for every $f \geqq 0$.

Clearly the condition is necessary. Now if $P f \geqq \alpha P_{1} f$ for every $f \geqq 0$ define $P_{2} f=(1-\alpha)^{-1}\left(P f-P_{1} f\right)$. Then $P_{2}$ satisfies (1.2) and (1.3). In order to prove (1.1) it is enough to observe that $P_{2}$ is a contraction on $L_{\infty}$ and if $f=\Sigma c_{i} 1_{A_{i}}$, where $A_{i}$ are disjoint sets and $1_{A_{i}}$ denote their characteristic functions, then

$$
\int\left|P_{2} f\right| d \lambda \leqq \Sigma\left|c_{i}\right|(1-\alpha)^{-1} \int\left(P-\alpha P_{1}\right) 1_{A_{i}} d \lambda=\Sigma\left|c_{i}\right| \lambda\left(A_{i}\right)
$$

since

$$
\begin{aligned}
\int\left(P-\alpha P_{1}\right) 1_{A_{i}} d \lambda & =\left\langle\left(P-\alpha P_{1}\right) 1_{A_{i}}, 1\right\rangle=\left\langle 1_{A_{i}},\left(P^{*}-\alpha P_{1}^{*}\right) 1\right\rangle \\
& =(1-\alpha)\left\langle 1_{A_{i}}, 1\right\rangle=(1-\alpha) \lambda\left(A_{i}\right),
\end{aligned}
$$

where $\langle f, g\rangle$ is the inner product of $f$ and $g$.

3. Integral averages. Following Choquet's theory let us consider, throughout this chapter, the following setup:

(3.1) Let $\mu$ be a regular positive measure, of total mass 1, defined on the Borel subsets of $\mathfrak{A}$ with its weak operator topology. Put

$$
Q=\int_{\mathscr{R}} P \mu(d P) .
$$

The operator $Q$ is defined by $\langle Q f, g\rangle=\int_{\mathfrak{A}}\langle P f, g\rangle \mu(d P)$. The integral exists since for every pair of vectors $f, g$ the function $\phi(P)=\langle P f, g\rangle$ is continuous in the weak operator topology. Thus $\int \mathfrak{x}\langle P f, g\rangle \mu(d P)$ defines a bilinear form and hence is equal to $\langle Q f, g\rangle$ for some operator $Q$. It is easy to check that $Q$ belongs to $\mathfrak{A}$. 
Let us consider all the open subsets of $\mathfrak{A}$ on which $\mu$ vanishes. Since $\mu$ is regular $\mu$ vanishes also on the union of all these sets. Denote by $\mathfrak{B}$ (support of $\mu$ ) the complement of this set. Thus

(3.2) $P_{0} \in \mathfrak{B}$ iff $\mu$ does not vanish on any neighborhood of $P_{0}$.

Theorem 3. Given $Q$ by (3.1) then

$$
K(Q)=\bigcap_{P \in \mathscr{B}} K(P) \cap \bigcap_{P \in \mathscr{B}}\left\{f: P^{n} f=Q^{n} f, P^{* n} f=Q^{*_{n}} \text { for all } n\right\} .
$$

Proof. Let $f \in K(Q)$ and $P_{0} \in \mathfrak{B}$ and let $\mathfrak{D}$ be any weak neighborhood of $P_{0}$. Put

$Q=\mu(\mathfrak{D})\left(\mu(\mathfrak{D})^{-1} \int_{\mathfrak{D}} P \mu(d P)\right)+\mu(\mathfrak{B}-\mathfrak{D})\left(\mu(\mathfrak{B}-\mathfrak{D})^{-1} \int_{\mathfrak{B} \_\mathbb{D}} P \mu(d P)\right)$.

Then, by Theorem $1,\left(\mu(\mathfrak{D})^{-1} \int_{\mathfrak{D}} P \mu(d P)\right)(f)=Q f$. Or, for any $g \in L_{2}$, $\mu(\mathfrak{D})^{-1} \int_{\mathcal{D}}\langle P f, g\rangle \mu(d P)=\langle Q f, g\rangle$. If for some $g\left\langle P_{0} f, g\right\rangle \neq\langle Q f, g\rangle$ say $\left\langle P_{0} f, g\right\rangle\langle\langle Q f, g\rangle$ then taking for $\mathfrak{D}$ the open set $\{P:\langle P f, g\rangle\langle\langle Q f, g\rangle\}$ we shall get a contradiction. Thus for every $P \in \mathfrak{B}, P f=Q f$. Now $P^{n} f=P^{n-1} Q f$ and $Q f \in K(Q)$ too and by an induction argument $P^{n} f=Q^{n} f$. The argument for $P^{*}$ is analogous.

Conversely, if $f$ belongs to the right side of (3.3) then take any $P$ in $\mathfrak{B}$ and $\left\|Q^{n} f\right\|=\left\|P^{n} f\right\|=\|f\|$, since $f \in K(P)$.

REMARK. Theorem 3 can be viewed as a necessary condition for an element $P$ of $\mathfrak{A}$ to belong to the support of any representation of the type (3.1).

Theorem 4. Given $Q$ by 3.1 then

$$
H_{1}(Q)=K(Q) \cap \bigcap_{P \in \mathscr{B}} H_{1}(P) .
$$

The proof is identical to the proof of Theorem 2 .

4. Semigroup of contractions. Let us conclude this note with a study of convergence of iterates of the resolvent of a semigroup of contractions. The situation is somewhat similar to the one studied in Chapter 3 but much stronger results are valid.

Let $P_{t}$ be a strongly continuous semigroup of contractions in the Hilbert space $H$. Let $R_{\lambda}=\int_{0}^{\infty} e^{-\lambda t} P_{t} d t, \lambda>0$. Thus $R_{\lambda}, \lambda>0$, is the resolvent of the infinitesimal generator, $A$, of $P_{t}$ at the point $\lambda$. Let $U_{t}$ be the strong dilation of the semigroup see [4, Theorem IV]. Then $U_{t}$ is a strongly continuous semigroup of unitary operators. Let the infinitesimal generator of $U_{t}$ be $i B$. Then $B$ is selfadjoint [5, p. 385]. Thus 


$$
\int_{0}^{\infty} e^{-\lambda t} U_{t} d t=(\lambda-i B)^{-1} \quad \lambda>0 .
$$

The spectrum of $\lambda(\lambda-i B)^{-1}$ is included in $\left\{\lambda(\lambda-i t)^{-1}: t\right.$ is real $\}$. This set is inside the unit circle and touches the circumference of the unit circle at the point 1 only. Now $\lambda(\lambda-i B)^{-1}$ is a normal operator and, from the Spectral Theorem and the above description of the spectrum, follows that $\left(\lambda(\lambda-i B)^{-1}\right)^{n} f$ is strongly convergent for every $f$ in the larger space where the $U_{t}$ are defined.

THEOREM 5. Let $R_{\lambda}=\int_{0}^{\infty} e^{-\lambda t} P_{t} d t, \lambda>0$. Then $\lim \left(\lambda R_{\lambda}\right)^{n} f=$ projection of $f$ on the set $\left\{g: P_{t} g=g\right.$ for all $\left.t>0\right\}$.

Proof. Let $L=\left\{g: P_{t} g=g\right.$ for all $\left.t\right\}$ and $f=f_{1}+f_{2}$ where $f_{1} \in L$ and $f_{2} \perp L$. Clearly $\lambda R_{\lambda} f_{1}=f_{1}$ and we shall consider $f_{2}$ only. Now $\left\|P_{t}\right\| \leqq 1$ and thus $P_{t} g=g$ if and only if $P_{t}^{*} g=g$ or $L^{\perp}$ is invariant under $P_{t}$. Now

$$
\left(\lambda R_{\lambda}\right)^{n}=\lambda^{n} \int_{0}^{\infty} \cdots \int_{0}^{\infty} e^{-\lambda\left(t_{1}+\cdots+t_{n}\right)} P_{t_{1}+\cdots+t_{n}} d t_{1} \cdots d t_{n}
$$

and is equal to the projection of $\left[\lambda(\lambda-i B)^{-1}\right]^{n}$ on $H$. Thus $\left(\lambda R_{\lambda}\right)^{n} f_{2}$ is strongly convergent too. Let its limit be $h$. Then $\lambda R_{\lambda} h=h$. Hence $h$ belongs to the domain of definition of $A$ and $(A-\lambda) h=\lambda(A-\lambda) R_{\lambda} h$ $=\lambda h$ or $A h=0$. Thus $h \in L$ but $f_{2}$ and $\left(\lambda R_{\lambda}\right)^{n} f_{2}$ belong to $L$. Therefore $h=0$.

\section{REFERENCES}

1. S. R. Foguel, The ergodic theorem for Markov processes, Israel J. Math. 4 (1966), 11-22.

2. - Invariant subspaces of a measure preserving transformation, Israel J. Math. 2 (1964), 198-200. 1956.

3. P. R. Halmos, Lectures on ergodic theory, The Mathematical Society of Japan,

4. B. Sz.-Nagy, Prolongements des transformations de l'espace de Hilbert qui sortent de cet espace (Appendice au livre Leçons d'analyse fonctionelle), Akadémiai Kiad6, Budapest, 1955.

5. F. Riesz and B. Sz.-Nagy, Functional analysis, Ungar, New York, 1955.

The Hebrew University of Jerusalem and

University of Minnesota, Minneapolis 